\title{
In Vitro Analysis of the Effects of Two Air-Abrasive Prophylaxis Systems and Inlet Air Pressure on the Surface of Titanium Abutment Cylinders
}

\author{
Michael E. Razzoog, DDS, MS, MPH, ${ }^{*}$ and Sreenivas Koka, DDS, MS†
}

\begin{abstract}
Purpose: The purpose of this study was to investigate the effects of two air-abrasive prophylaxis systems and the effect of inlet air pressure on the surface of Branemark titanium abutment cylinders.

Materials and Methods: Single abutment cylinders were treated with either the Prophy-Jet system (sodium bicarbonate abrasive) (Dentsply International, York, PA) or the Microprophy system (aluminum oxide abrasive) (Danville Engineering $\mathrm{Co}$, Danville, CA) for 60 seconds at an inlet air pressure of 60 psi or 90 psi. The effects on the surface of each abutment cylinder were visually inspected by scanning electron microscopy.

Results: A comparison of abutment cylinder surfaces after treatment showed that the ProphyJet system removed machining marks to a greater degree than the Microprophy system. Sodium bicarbonate particles from the Prophy-Jet system were significantly larger than the aluminum oxide particles used with the Microprophy system, potentially accounting for the difference in abrasivity. In addition, inlet air pressure of 60 psi caused removal of machining marks to a greater degree than an inlet air pressure of 90 psi. The principle of phase separation may account for the lower inlet air pressure causing more removal of machining marks than the higher inlet air pressure.

Conc/usions: Under the experimental conditions tested, neither of the two systems tested seemed to cause significant abrasion of the surface of titanium abutment cylinders.
\end{abstract}

J Prosthod 1994;3: 103-107. Copyright 1994 by the American College of Prosthodontists.

INDEX WORDS: titanium, prophylaxis, air abrasive, dental implants

A $S$ THE NUMBER of patients receiving treatment with osseointegrated dental implants increases, the long-term maintenance of dental implants should concern the dental profession. Preliminary data indicate that the peri-implant soft tissue response to plaque is similar to that found in gingivitis associated with teeth, and peri-implant inflammation of soft tissue seems to be directly related to plaque accumulation. ${ }^{1}$ One method that claims to clean and polish the supragingival titanium surface of osseointegrated dental implants is an air-powder abrasive system. The potential for abrasion of the titanium surface by using such a technique has been a serious concern. ${ }^{24}$ Preliminary documentation of visual data of the effect of one

*Associate Professor, Department of Prosthodontics, University of Michigan, School of Dentistn; Ann Arbor, MI.

TAssistant Professor, Department of Adull Restorative Dentistry, University of Nebraska Medical Center, College of Dentisity, Lincoln, NE.

Correspondence to: Sreenivas Koka, DDS, MS, College of Dentisty, University of Nebraska Mediual Center, 40th and Holdredge, Lincoln, NE. 68583-0740.

Copyright 01991 by the American College of Prosthodontisis

$1059-941 \times 19410302-0010 \$ 5.0010$ air-powder abrasive system (Prophy-Jet, Dentsply International, York, PA), which uses sodium bicarbonate as the abrasive, on the surface of machined titanium has been reported. ${ }^{5}$ The results of this study suggest that an air-abrasive system may remove machining marks from Brånemark titanium abutment cylinders. Recently, a new air-abrasive device intended to aid practitioners and hygienists in the removal of plaque and calculus from implant surfaces (Microprophy, Danville Engineering Co, Danville, CA) has becn introduced. The Microprophy system offers the practitioner a choice of two abrasives for use with the system, either aluminum oxide or sodium bicarbonate. However, the effect of aluminum oxide when used as an air-abrasive prophylaxis agent on the surface of machincd titanium has not been reported.

Variation of air pressure affects the abrasivity of an air-abrasive prophylaxis system, with a higher air pressure expected to cause more abrasion than a lower air pressure. ${ }^{6}$ The manufacturers of both the Prophy-Jet and Microprophy air-abrasive prophylaxis systems suggest that an inlet air pressure of 60 psi to 90 psi be used with their system. However, the effect 
of varying inlet air pressure on the abrasivity of air-powder abrasive prophylaxis systems on machined titanium has not been documented.

The purposes of the present study were as follows:

1. To compare the effect on the surface of machined titanium of an air-abrasive system, which relies on sodium bicarbonate as its abrasive medium, with the effect on the surface of machined titanium of an air-abrasive system that uses aluminum oxide as its abrasive medium.

2. To compare the effect of inlet air-pressure on the abrasivity of two different air-abrasive systems.

All evaluation was performed visually by means of scanning electron microscopy (SEM).

\section{Materials and Methods}

Five Brånemark abutment cylinders (Nobclpharma, Nobel Industries, Gothenburg, Sweden) were individually fixed with Duralay resin (Reliance Dental, Worth, IL) to a custom-made aluminum base that could be removed and re-inserted into a separate Duralay receptaclc. To maintain consistent exposure conditions, a custom-designed aluminum jig was fabricated that ensured delivery of spray from an angle of $60^{\circ}$ to the abutment cylinder surface, and from a distance of $5 \mathrm{~mm}$, according to the manufacturer's recommendations. An air-pressure regulator (A-Dec, Newberg, OR) was used to ensure that inlet air pressure was maintained at the desired experimental levels.

The Prophy-Jet and Microprophy systems were assembled according to manufacturer's recommendations and the abutment cylinders treated as follows:

- Cylinder no. 1: No treatment. This cylinder was used as a control.

- Cylinder no. 2: Exposure to the Prophy-Jet for 60 seconds at an inlet air pressure of 60 psi.

- Cylinder no. 3: Exposure to the Microprophy for 60 seconds at an inlet air pressure of 60 psi.

- Cylinder no. 4: Exposure to the Prophy-Jet for 60 seconds at an inlet air pressure of 90 psi.

- Cylinder no. 5: Exposure to the Microprophy for 60 seconds at an inlet air pressure of 90 psi.

Before SEM analysis, all the cylinders were rinsed with distilled water for 10 seconds and allowed to dry.

The cylinders were glucd on an aluminum specimen mount using conductive glue and examined with an AMRAY 1000-B scanning electron microscope (AMRAY Inc, Bedford, MA). In addition, $0.25 \mathrm{~g}$ of Prophy-Jet cleaning powder- 1 and $0.1 \mathrm{~g}$ of Microprophy aluminum oxide abrasive powder were separately examined by SEM to determine modal particle size.

\section{Results}

\section{Scanning Electron Microscopy}

SEM $(1,000 \times)$ of the untreated abutment cylinder showed machining marks in the forms of subtle ridges and grooves (Fig 1). Debris from localized contamination during mounting also appeared with minimal damage to the machining marks (not shown).

When SEM (1,000x) of the abutment cylinder treated with sodium bicarbonate at 60 psi were compared with the abutment cylinder treated with aluminum oxide at 60 psi, treatment with sodium bicarbonate led to a greater degree of removal of the machining marks (Figs 2 and 3). A similar difference between the two systems was observed at an air pressure of 90 psi (Figs 4 and 5).

When SEMs $(1,000 \times)$ of the abutment cylinders treated with the same system but using different air pressures were evaluated, surfaces treated at 90 psi were less affected than those treated at 60 psi. (Compare Fig 2 with Fig 4, and Fig 3 with Fig 5.) SEMs of the abrasive powders arc presented in Figures 6 and 7.

\section{Discussion}

Air-powder abrasive prophylaxis systems have increased in popularity as a method for removing calculus and plaque from natural teeth. (For an overview of the principles associated with the use of air-powder abrasive prophylaxis systems, the reader is referred to Koka et al.7) However, they should be used with caution and carc. Gingival trauma caused by desquamation of the epithelial layer has been

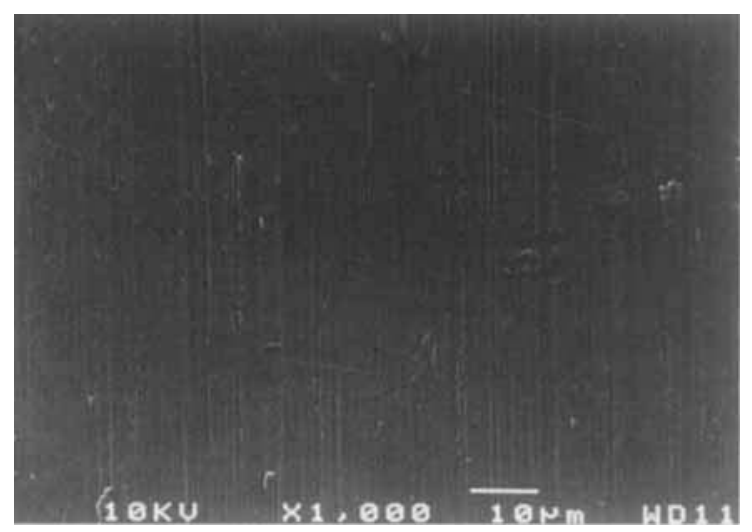

Figure 1. SEM of the untreated abutment cylinder $(1,000 \times)$. 


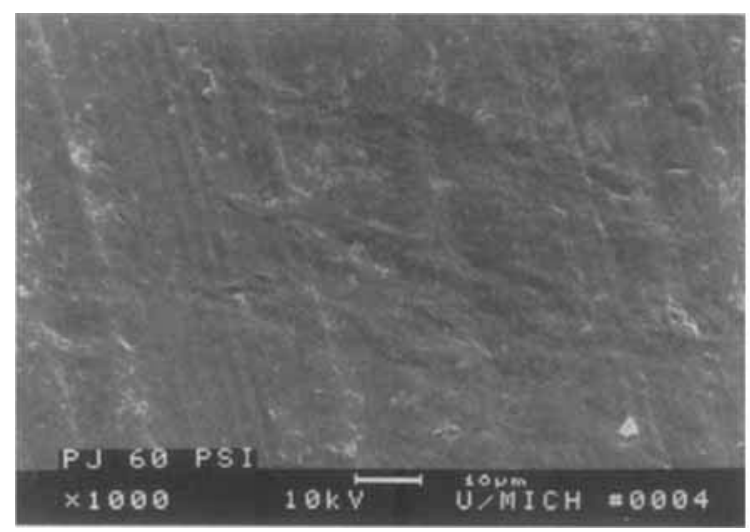

Figure 2. SEM of the abutment cylinder treated with sodium bicarbonate at $60 \mathrm{psi}(1,000 \times)$.

reported associated with the indiscriminate use of the Prophy-Jet system, and care should be taken to cnsure that the nozzle tip is not directed toward soft tissue ${ }^{8-10}$ In addition, a reported case of pneumoparotitis highlights the risk of air embolism formation secondary to placcment of the nozzle tip into the gingival sulcus. "

Variables such as water flow, exposurc time, configuration of specimen, angulation of tip to specimen, lubricant, frictional coefficient of the specimen, and nozzle-target distance may affect the abrasivity of systems such as those used in this study. In addition, other factors such as the particle hardness, speed of the particle, size of the particle, and air pressure may also influcnce the abrasivity of airpowder abrasive prophylaxis devices. ${ }^{6}$

Previous data suggest that use of the Prophy-Jet system, which uses sodium bicarbonate particle sizes greater than $30 \mu \mathrm{m}$, may lead to complete removal

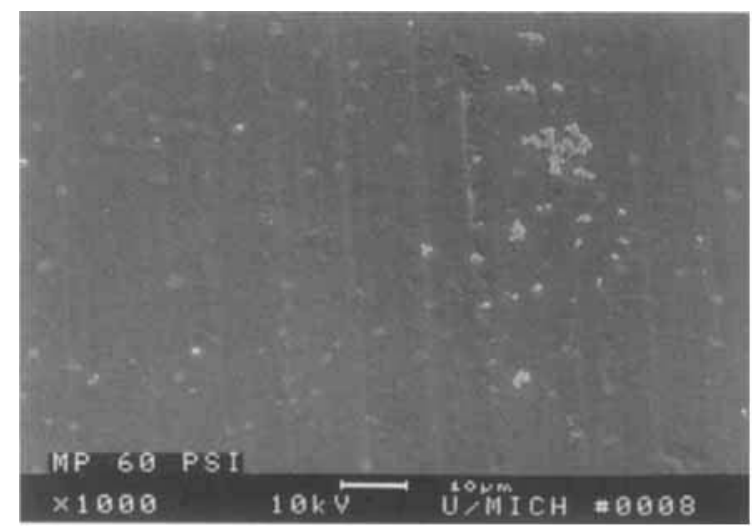

Figure 3. SFM of the abutment cylinder treated with aluminum oxide at $60 \mathrm{psi}(1,000 \times)$.

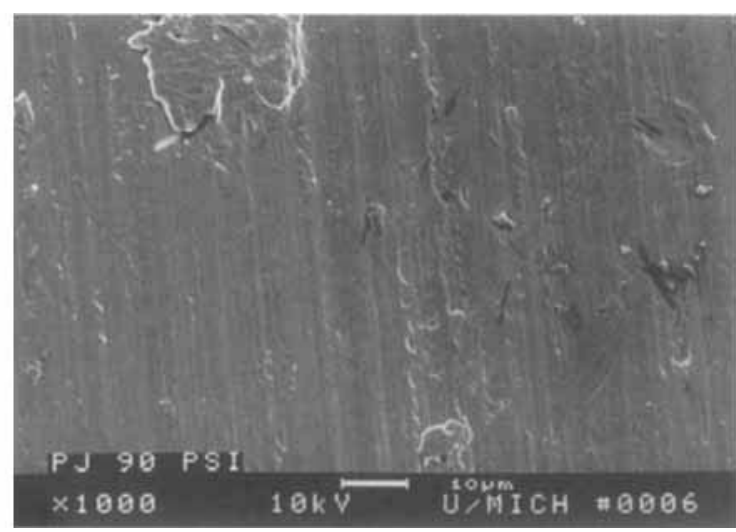

Figure 4. SEM of the abutment cylinder treated with sodium bicarbonate at 90 psi $(1,000 \times)$.

of the machining marks from a Brannemark titanium abutment cylinder. ${ }^{7}$ However, in this study, complete removal of machining marks was not observed. The discrepancy may be due to the fact that the duration of exposure to the air-abrasive spray was shorter in this study.

SEM analysis displaying machining marks on the surface of the control abutment cylinder corroborated previously published data. 5,7 In this study, comparison of the effects of an air-powder abrasive prophylaxis system that uses sodium bicarbonate abrasive particles, with an air-powder abrasive prophylaxis system that uses aluminum oxide particles showed that the former secmed to remove machining marks to a greater degrec when used at the same air-pressure. Sizes of sodium bicarbonate particles ranged from $40 \mu \mathrm{m}$ to $100 \mu \mathrm{m}$ (Fig 6), but the aluminum oxide particles were significantly smaller, less than $1 \mu \mathrm{m}$ in diameter (Fig 7 ). Smaller particles

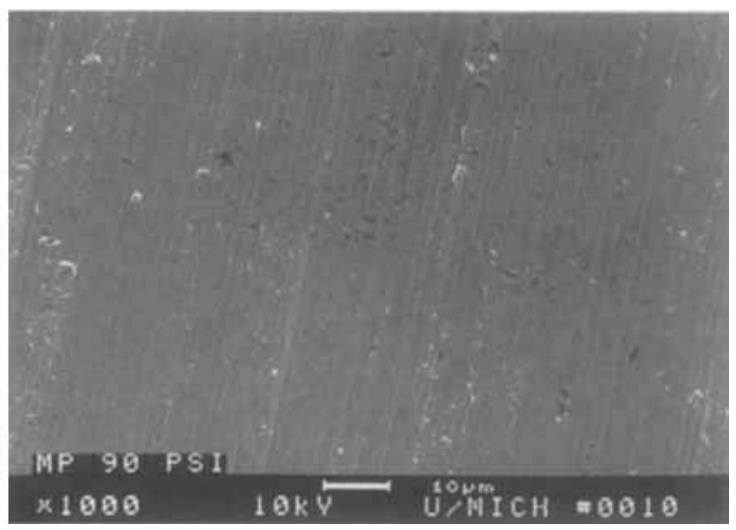

Figure 5. SEM of the abutment cylinder treated with aluminum oxide at $90 \mathrm{psi}(1,000 \times)$. 


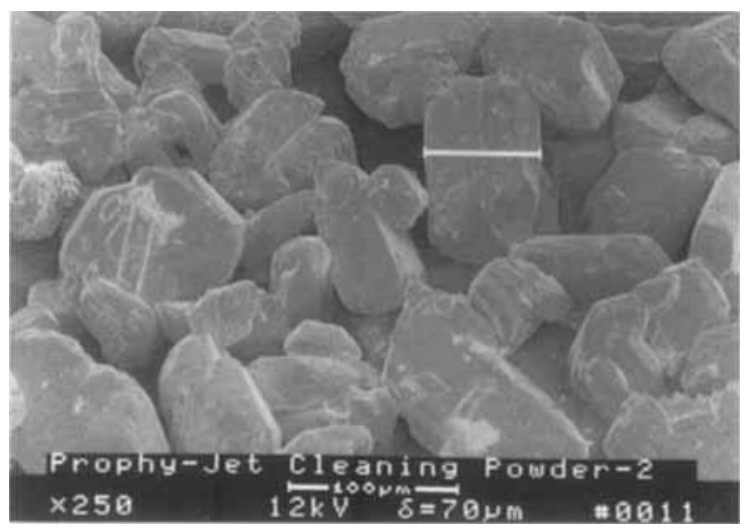

Figure 6. SEM of sodium bicarbonate abrasive powder $(250 \times)$.

would be expected to cause less abrasion than larger particles, perhaps accounting for the difference in surface alteration.

A comparison of the effect of altering air pressure was surprising because the lower air pressure investigated (60 psi) seemed to cause increased removal of machining marks than the higher air pressure (90 psi). Of the four experimental treatments, the use of the Microprophy system at 90 psi showed the least effect when compared with the control surface. An answer to this apparent paradox may lie in the mechanical engineering field of fluid dynamics, in particular one-dimensional, two-phase flow. ${ }^{12}$ The mixture of abrasive particles and water is expelled through a narrow nozzle tip. In general, the mixture flows out through the minimum cross-sectional area of the nozzle at a pressure $p$. The mass flow (or mass rate of flow), $m$, at any point along the nozzle is given by the equation of continuity ( $A=$ cross-scctional area, $\mathrm{V}=$ velocity):

$$
m=p \mathrm{VA}
$$

Additional analysis allowing for the substitution of $\mathrm{V}$ using the Bemoulli equation shows that as the pressure increases, the mass flow increases. However, this is a general observation that is only valid at pressures less than a certain pressure referred to as the critical pressure. The critical pressurc relates to the pressure at which maximum mass flow occurs. It seems that at pressures either more than or less than the critical pressure, the mass flow will be lower than that obscrved at the critical pressure, ie, above the critical pressure less mass flow is possible than at a lower pressure. This phenomenon is known as chaked flow.$^{13}$ For a more in-depth analysis of one-dimen- sional, two-phase flow and the principle of choked flow, the reader is referred to Wallis. ${ }^{12}$

The study's results suggest that an air pressure of $90 \mathrm{psi}$ is above the critical pressure due to the lesser degree of removal of machining marks observed (less mass flow of abrasive particles). Unfortunately, because an estimate of the critical pressure is impossible from our data, we arc unsure of whether 60 psi is above or below the critical pressure. Further research with differing air pressures would be necessary to determine the critical pressure. However, of more clinical relevance is the following question: "At what inlet air pressurc is plaque and calculus efficiently removed?"

Although there has been concern about the risk of excessive abrasion caused by air-powder abrasive prophylaxis devices, the present study indicates that exposure to cither the Prophy-Jet system with sodium bicarbonate as the abrasive or the Microprophy system with aluminum oxide as the abrasive does not lead to significant surface alteration of a titanium abutment cylinder surface, especially when an air pressure of $90 \mathrm{psi}$ is used. These findings confirm those of Homiak et $\mathrm{al}^{5}$ and show significantly less surface alteration to titanium than that observed with metal scalers, but slightly more surface alteration than that observed with the use of plastic scalers after 30 strokes with the respective prophylaxis instruments. However, one should realize that the resultant surfaces in this study were rcpresentative of 60 seconds of exposure, a period of time that equates to 12 prophylaxes on the assumption that any one area of an abutment cylinder is exposed to the abrasive spray for an averagc of 5 seconds per prophylaxis. The fact that the machining marks were still evident after 60 seconds of treatment suggests

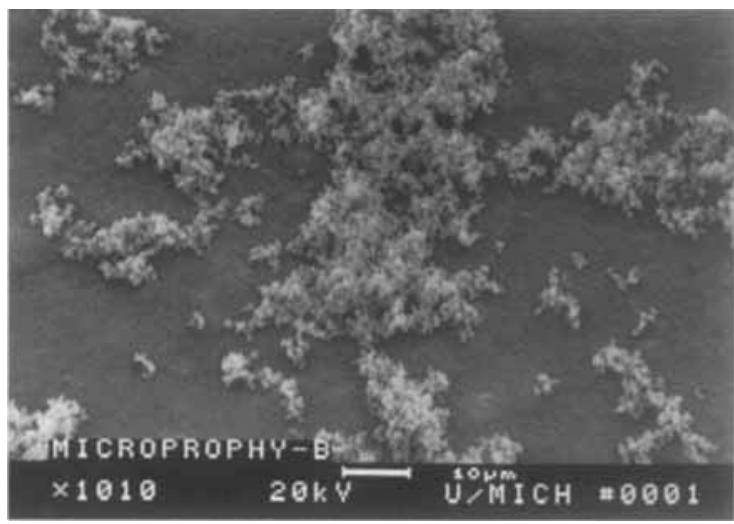

Figure 7. SEM of aluminum oxide abrasive powder $(1,010 \times)$. 
that any abrasion and subsequent loss of titanium is minimal. Although the greatest surface alteration was observed with sodium bicarbonate particles at an air pressure of $60 \mathrm{psi}$, the machining marks are still visible.

The present study concentrated on the in vitro effects of two differcnt air-powder abrasive prophylaxis systems and two different inlet air pressures on the surface of machined titanium. In vivo effects were not evaluated, and the relative efficiencies of the different experimental conditions in terms of plaque removal from abutment cylinders and subsequent plaque adherence to the same cylinders have not been reported. It is possible that an interceding layer of plaque between the abrasive spray and the abutment cylinder may lead to surface alteration of titanium that differs from that observed in this study where uncontaminated titanium was used. Further study to determine the effectivencss under controlled clinical conditions of air-powder abrasive prophylaxis systems, materials, and conditions of use is indicated.

\section{Conclusions}

Neither of the tested systems caused significant abrasion of the surface of Brånemark titanium abutment cylinders. The Prophy-Jet system, which uscd sodium bicarbonate particles (40 to $100 \mu \mathrm{m}$ ) as an air-powder prophylaxis abrasive, seemed to cause more surface alteration of a titanium abutment cylinder than the Microprophy system, which used aluminum oxide particles $(<1 \mu \mathrm{m})$. The use of an inlet air pressure of 90 psi seemed to cause less surface alteration of the surface of a Brancmark titanium abutment cylinder than the use of an inlet air pressure of 60 psi. The phenomenon of choked flow is suggested as a cause for this apparent paradox.

\section{References}

1. Albrektsson T, Zarb GA: The Brånemark Osscointegrated Implant. Chicago, IL, Quintessence, 1989

2. Atkinson DR, Cobb CM, Killoy WJ: The effect of an airpowder abrasive system on in-vitro root surfaces.J Periodontol 1984:55:13-18

3. Petersson LG, Hellden L, Jongebloed W, et al: The effect of a jet abrasive instrument (Prophy Jet) on root surfaces. Swed Dent J 1985;9:193-199

4. Lubow RM, Cooley RL: Effect of air-powder abrasive instrument on restorative materials. J Prosthet Dent 1986;55:462465

5. Homiak AW, Cook PA, DeBoer J: Efrect ol hygiene instrumentation on titanium abut ments: a scanning elect ron microscopy study. J Prosthet Dent 1992;67:364-369

6. Waimsley AD, Williams AR, Laird WRE: The air-powder dental abrasive unit-an evaluation using a model system. J Oral Rehabil 1987;14:43-50

7. Koka S, Han JS, Razzoog MF, ct al: The effects of two air-powder abrasive prophylaxis systems on the surface of machined titanium: A pilot study. Implant Dent 1992;1:259265

8. Mishkin DJ, Engler WO, Javed T, et al: A clinicat comparison of the effect on the gingiva of the Prophy-Jet and the rubber cup and paste techniques.J Periodontol 1986;57:151-154

9. Newman PS, Silverwood RA, Dolby AE: The effects of an air-abrasive instrument on dental hard tissues, skin and oral mucosa. Br Dent J 1985; 159:9-12

10. Kontturi-Narhi V, Markkanen S, Markkanen H: The gingival effects of dental airpolishing as evaluated by scanning electron microscopy. J Periodontol 1989;60:19-22

11. Brown FH, Ogletree RC, Houston GD: Pneumoparotitis associated with the use of an air-powder prophylaxis unit. J Periodontol 1992;63:642-644

12. Wallis GB: One-Dimensional Two-Phase Flow. New York, NY, McGraw-Hill, 1969

13. Yuan SW: Foundations of Fluid Mechanics. Englcwood Cliffs, NJ, Prentice-Hall, 1967 